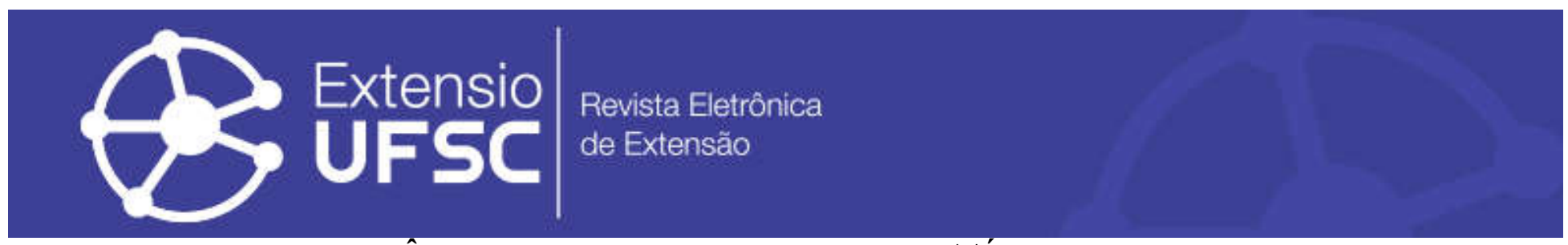

\title{
UMA VIVÊNCIA BRASIL-COLOMBIA EM ESTÁGIO DE ENFERMAGEM
}

Michaela Byron Correa dos Santos Universidade Federal do Estado do Rio de Janeiro mbyroncs@gmail.com

\author{
Emanuel Pereira dos Santos \\ Hospital Universitário Gaffrée e Guinle \\ emanuelgranarcanjo@gmail.com
}

Lina Maria Casas Ramirez Universidad Católica de Manizales lina.casas@ucm.edu.com

\section{Resumo}

Trata-se relato de experiência. Objetivo: Relatar a experiência de estudantes do curso de graduação em Enfermagem da Universidade Federal do Estado do Rio de Janeiro e da Universidade Católica de Manizales, no contexto dos programas de mobilidade estudantil. Métodos:01 Estudante de Enfermagem - UCM Colômbia; 02 estudantes de Enfermagem EEAP/UNIRIO e 01 professora de Administração da EEAP/UNIRIO; 01 enfermeiro-chefe da enfermaria pediátrica. Período de Estágio Curricular Obrigatório em Administração em Enfermagem, entre agosto e setembro de 2019, em um total de 150 horas. Resultados: As atividades realizadas visaram à melhoria da qualidade assistencial em enfermagem: solicitação de material, conservação e organização para o completo funcionamento da enfermaria, treinamento de equipe e aplicação de escalas de rotina. As atividades tiveram êxito e fornecerão subsídios para futuras publicações. Conclusão: Os estudantes puderam trocar experiências e obtiveram conhecimento de língua estrangeira, cultura, diferenças dos sistemas de saúde e de ensino. A experiência se mostrou benéfica.

Palavras-chave: Educação em Enfermagem. Intercâmbio Internacional Educacional. Diplomacia em Saúde.

\section{EXPERIENCE BRAZIL-COLOMBIA IN NURSING INTERSHIP}

\begin{abstract}
This is an experience report. Objective: To report the experience of undergraduate Nursing students from the Federal University of the State of Rio de Janeiro and the Catholic University of Manizales, in the context of student mobility programs. Methods: 01 Nursing student - UCM Colombia; 02 EEAP / UNIRIO Nursing students and 01 Administration professor at EEAP / UNIRIO; 01 head of pediatric nursing. Compulsory Curricular Internship in Nursing Administration, between August and September 2019 in a total of 150 hours. Results: The activities carried out were related to improving the quality of nursing care: request for material, custody and organization for the complete operation of the nursing unit, team training and application of routine scales. The activities were successful and will provide grants for future publications. Conclusion: The students were able to exchange experiences and obtained knowledge of foreign language, culture, differences in health and education systems. The experience was beneficial.
\end{abstract}

Keywords: Education Nursing. International Education Exchange. Health Diplomacy.

\section{UNA EXPERIENCIA BRASIL-COLOMBIA EN ETAPA DE ENFERMERÍA}

\section{Resumen}

Este es un informe de experiência. Objetivo: Informar la experiencia de estudiantes de pregrado en Enfermería de la Universidad Federal del Estado de Río de Janeiro y la Universidad Católica de Manizales, en el contexto de programas de movilidad estudiantil. Métodos: 01 Estudiante de Enfermería - UCM Colombia; 02 estudiantes de Enfermería EEAP / UNIRIO y 01 profesor de Administración en EEAP / UNIRIO; 01 jefa de enfermería de pediatría. Pasantía Curricular Obligatoria em Administración de Enfermería, entre agosto y septiembre de 2019 en un total de 150 horas. Resultados: Las actividades realizadas estuvieron relacionadas com la mejora de la calidad de la atención en enfermería: solicitud de material, custodia y organización para el completo funcionamiento de la enfermería, formación del equipo y aplicación de escalas de rutina. Las actividades tuvieron éxito y proporcionarán subsidios para futuras publicaciones. Conclusión: Los Estudiantes obtuvieron conocimientos de lengua extranjera, cultura, diferencias en los sistemas de salud y educación. La experiência resultó beneficiosa.

Palavras clave: Educación en Enfermería. Intercambio Internacional Educacional. Diplomacia en la Salud. 
Uma vivência Brasil-Colombia em estágio de Enfermagem

\section{INTRODUÇÃO}

Sabendo que "profissionais da saúde necessitam de consciência e entendimento sobre como a cultura influencia a saúde e crenças de saúde" (CARLSON, et al., 2017, p.54) e em sintonia com a crescente globalização e política de abertura de fronteiras, havendo assim numerosos imigrantes dos mais diversos países, a internacionalização do ensino mostra-se importante. "A mobilidade acadêmica internacional oferece um leque de oportunidades para a expansão dos conhecimentos científicos e visões de mundo, proporcionando ao estudante uma visão ampla, reflexiva e crítica acerca das questões sociais, políticas, econômicas, pessoais e profissionais" (GUSKUMA, et al., 2016, p. 989), de forma a conseguir preparar o profissional da saúde, por exemplo, para o cuidado com populações diversas.Portanto, para a promoção de políticas de internacionalização de ensino, alguns governos criam medidas de incentivo ao intercâmbio acadêmico.Para conhecer alguns aspectos da Colômbia e do Brasil,os dois países cujos estudantes participaram deste intercâmbio de experiências em Enfermagem, aborda-se a seguir, brevemente, características de cada um em três campos:na cultura, educação e saúde.

\section{Cultura:}

Devido à sua história colonial, a cultura colombiana é muito semelhante à de outros países da América Latina. O legado colonial dos espanhóis se estende por todo o país e os povos indígenas se distanciaram de suas raízes pré-colombianas. Hoje, a cultura colombiana é uma mistura de costumes e tradições européias e indígenas que se refletem na música, na arte, na literatura e no relacionamento com a natureza.

A cultura do Brasil é uma síntese da influência dos diversos povos e grupos étnicos que formaram a população brasileira. Não existe uma cultura brasileira perfeitamente homogênea, mas um mosaico de diferentes aspectos culturais que, juntos, formam a cultura do Brasil. Evidentemente, depois de três séculos de colonização portuguesa, até a independência do país, a cultura do Brasil adquiriu fortes raízes lusitanas, bem como de correntes migratórias posteriores, vindas de todos os continentes, somadas à intensa miscigenação. O Brasil é composto por povos diferentes, com reflexo em sua cultura, em que se incluem principalmente elementos indígenas, africanos e europeus, estes últimos de origem portuguesa, cuja língua é o português.

\section{Educação:}

Para entender a abrangência do conceito de educação, vale destacar o primeiro parágrafo da Lei no 9.394, de 20 de dezembro de 1996, que estabelece as diretrizes da educação 
Uma vivência Brasil-Colombia em estágio de Enfermagem

brasileira, abrangendo "processos formativos que se desenvolvem na vida familiar, na convivência humana, no trabalho, nas instituições de ensino e pesquisa, nos diversos movimentos e organizações da sociedade civil e nas manifestações culturais". É nesse sentido que é importante pensar os currículos de Enfermagem a seguir. O currículo de graduação em Enfermagem da Universidade Católica de Manizales busca proporcionar a seus alunos conhecimentos científicos e "sócio-bumanísticos", que sustentam a profissão, os princípios éticos e a sensibilidade social para liderar e gerenciar o cuidado para com as pessoas enquanto indivíduos, família, comunidade,e sempre a partir de uma visão interdisciplinar do entendimento do conceito de cuidado.É um currículo que também visa desenvolver a capacidade dos alunos para gerenciar e implementar políticas para a melhoria da qualidade de vida, para a promoção da saúde e prevenção de doenças - o que vai ao encontro das propostas curriculares da Universidade Federal do Estado do Rio de Janeiro (UNIRIO).

A Escola de Enfermagem Alfredo Pinto (EEAP) é a primeira Escola de Enfermagem do Brasil, tendo sido criada em 1890 e reinaugurada em 1905. Foi só em 1979 que ela passou a integrar a UNIRIO. Atualmente, o curso de graduação em Enfermagem na EEAP é formado por 10 períodos, dos quais o $9^{\circ}$ e $10^{\circ}$ períodos são exclusivos para cumprimento de Estágio Curricular Obrigatório e elaboração do Trabalho de Conclusão de Curso. Os estágios são separados por 6 campos, sendo eles: Psiquiatria, Atenção à Saúde do Adulto e do Idoso, Saúde Coletiva, Administração, Atenção à Saúde da Criança e Atenção à Saúde da Mulher. A carga horária exigida é de oito horas diárias, sendo 32 horas semanais e 150 totais por campo, concluindo, portanto, 900 horas de estágio no último ano de curso.

Os modelos com os quais a UCM e a UNIRIO realizam suas práticas são os mesmos nos dois últimos semestres, em que os alunos cursam determinadas disciplinas,atuando na prática assistencial e administrativa de hospitais. Eles não têm um professor que os acompanha diariamente, mas precisam ter apresentado uma série de documentos para o hospital e para a universidade no semestre anterior. O aluno precisa, a partir daí, cumprir com um determinado número de horas para ser aprovado no curso, ganhando autonomia para escolher os dias em que vai atuar. A UNIRIO valoriza a realização de pesquisas e publicações por parte de seus professores, orientadores e alunos, em diferentes temas e áreas da Enfermagem, o que resulta em uma grande variedade de pesquisas realizadas ao longo de cada ano. Já a UCM ainda está caminhando em direção à pesquisa. As duas universidades têm em comum, na área de Enfermagem, a busca de uma formação com referências mais holísticas para os alunos. 
Uma vivência Brasil-Colombia em estágio de Enfermagem

\section{Saúde:}

O sistema geral de segurança social em vigor na Colômbia é regulado pela Lei 100, emitida em 23 de dezembro de 1993. A Colômbia em 2000 foi classificada em $22^{\circ}$ lugar dentre os 191 países citados no Relatório da Organização Mundial da Saúde, devido ao seu sistema geral de saúde.

O sistema de saúde colombiano caracteriza-se por ser composto por dois sistemas coexistentes: o regime contributivo (privado) e o regime subsidiado (gratuito), através do Sisbén (o que equivale ao serviço público de saúde no Brasil). Ambos os regimes oferecem cobertura universal, acesso igual a medicamentos, procedimentos cirúrgicos, serviços médicos e odontológicos.

O Ministério da Saúde e Proteção Social é responsável pelo desenvolvimento de políticas de saúde, bem como pela regulação da saúde no país. A Entidade Promotora de Saúde (EPS) é uma empresa regional de saúde responsável pela implementação dos objetivos estabelecidos pelo Ministério da Saúde, pelo desenvolvimento de linhas mestras e seus protocolos e também pela supervisão do desempenho dos cuidados de saúde.

Para cumprir suas responsabilidades, a EPS deve formar uma rede de serviços, de maneira que qualquer entidade promotora de saúde possa contratar clínicas, hospitais ou um Instituto de Saúde (IPS), de forma independente e autônoma, ou garantir o acesso a serviços com sua própria rede. Eles também devem contratar uma porcentagem mínima de instituições que prestam serviços públicos.

O sistema de saúde colombiano é descentralizado, as fontes de financiamento da saúde são principalmente do Sistema de Participação Geral (SGP), das contribuições de empregadores e trabalhadores ao regime contributivo administrado pelo Administrador dos Recursos do Sistema Geral de Seguridade Social em Saúde (ADRES), e dos recursos obtidos com jogos de sorte, como loterias, administrados por Coljuegos. Existem também outras fontes menores de financiamento (COLÔMBIA, 2019).

O Sistema Único de Saúde (SUS) do Brasil engloba todas as redes de atenção: Atenção Primária, de Média e Alta Complexidades, Serviços de Urgência e Emergência, Atenção Hospitalar, Assistência Farmacêutica, Vigilância Sanitária, Epidemiológica e Ambiental. O Sistema possui gestão participativa entre os três entes da Federação (União, Estados e Municípios) e proporciona o acesso gratuito, universal, integral, e sem discriminação, respeitando os princípios de universalidade, equidade e integralidade que regem o SUS (MS, 2019).

Este trabalho é um relato de experiência de interação entre estudantes e profissionais, com a presença de um estudante em intercâmbio, por meio do BRACOL, o programa de 
Uma vivência Brasil-Colombia em estágio de Enfermagem

intercâmbio de estudantes de graduação entre universidades do Brasil e da Colômbia. O objetivo principal é contribuir para o fortalecimento regional, por meio da aproximação acadêmicocientífica e cultural do corpo estudantil e profissional de ambos os países, promovido pela Associação Colombiana de Universidades (ASCUN) e por instituições associadas ao Grupo Coimbra de Universidades Brasileiras (GCUB), do Brasil.

A Universidade Federal do Estado do Rio de Janeiro recebeu, no segundo semestre de 2019, uma estudante colombiana para o Estágio Curricular de Administração em Enfermagem, localizado em uma enfermaria pediátrica de um Hospital Universitário, situado na Zona Norte do município do Rio de Janeiro. Nesse contexto, o artigo tem como objetivo, portanto, descrever a experiência de internacionalização de estudantes de Enfermagem nessa enfermaria pediátrica.

\section{MATERIAIS E MÉTODOS}

Este é um relato de experiência de internacionalização sobre a experiência e as atividades realizadas em um Hospital Universitário da Zona Norte do município do Rio de Janeiro, na enfermaria pediatria, por um estudante de enfermagem colombiano da Universidade Católica de Manizales, dois estudantes de Enfermagem da UNIRIO- Escola de Enfermagem Alfredo Pinto, um enfermeiro responsável pela enfermaria pediátrica e o professor da disciplina no segundo período do ano de 2019. A internacionalização do ensino superior habilita os dois países a promoverem meios de se incluírem e se desenvolverem. Assim, os alunos têm a oportunidade de viver juntos e aprender em novos ambientes acadêmicos, linguísticos e culturais, contribuindo com sua formação intelectual, profissional e humana, bem como com o desenvolvimento de sua competência intercultural (JUNG et al,, 2015, p.677). Neste caso, envolveu estudantes e profissionais do Brasil e da Colômbia.

Para realizar seus estudos no Brasil, foram solicitados os seguintes documentos e passos à estudante colombiana da referida experiência:

Possuir média geral de 4,0 (tendo em vista que as notas na Colômbia vão até, no máximo, 5). Escolher a universidade e o país onde o intercâmbio aconteceria; depois disso, encaminhar carta de garantia emitida ao conselho da faculdade, na qual os pais da estudante autorizam o processo de mobilidade. Na sequência, após aprovação da universidade, foi preciso comprovar níveis básicos de domínio do idioma (português). Estar em uma linha de pesquisa foi outro requisito. O Grupo de Comunicação em Saúde, por exemplo, tem a Linha Sorgen, que trabalha "a mobilidade de curar a si mesmo", contribuindo para a construção de novas posições e discussões acadêmicas do pensamento de Enfermagem, um importante produto da saúde pública 
Uma vivência Brasil-Colombia em estágio de Enfermagem

no contexto local, regional e nacional. Depois de preencher os formulários de validação de estudos nacionais e internacionais, com as respectivas cargas horárias e créditos de cada disciplina, realizaram-se exames psicotécnicos e médicos na UCM. AUNIRIO exige uma carta em que os alunos interessados expliquem por que decidiram realizar a mobilidade internacional na universidade brasileira, para, só assim,finalizar o processo. O passo final foi a confirmação por meio de Carta de Aceitação para realizar a mobilidade, para então se providenciar documentos solicitados pela UCM, como passaporte, visto de estudante, fotocópia da carteira de identidade e passagens aéreas. É possível obter desconto na taxa de inscrição.

As atividades realizadas pelos estudantes de Enfermagem, juntamente ao enfermeiro supervisor, durante o Estágio Obrigatório, foram referentes à administração, ao planejamento, à supervisão e à melhoria da qualidade assistencial em Enfermagem.São exemplos disso: a solicitação de material para o completo funcionamento da enfermaria, a conservação e organização desses materiais, o treinamento da equipe e a aplicação de escalas referentes à lesão por pressão e risco de queda para pacientes pediátricos.

As atividades em grupo para o acolhimento e bem-estar da estudante colombiana em intercâmbio incluíram conversas em espanhol nos primeiros dias (como forma de ensinar a ter empatia para com os estudantes) e reconhecimento do ambiente de estágio. O estágio aconteceu todos os dias úteis da semana, das 7 hàs $15 \mathrm{~h}$, entre a segunda quinzena de agosto e a primeira quinzena de setembro.

O que levou a aluna colombiana a realizar mobilidade acadêmica para o Brasil foi a percepção, através de pesquisa em artigos científicos, de que o Brasil detém grande conhecimento na área da Saúde, tendo em vista o volume desses artigos pesquisados ser em sua maioria brasileiros. Também foi importante o entendimento de que o intercâmbio cultural é ideal para melhorar as competências profissionais e pessoais. A experiência é adequada para desenvolver capacidades adaptativas a situações novas e complexas, de maneira a visualizar horizontes que permitam conhecer diferentes culturas e conviver com diferentes realidades.

A Escola de Enfermagem Alfredo Pinto da UNIRIO foi escolhida porque, ao se fazer uma pesquisa por universidades do Rio de Janeiro com uma carreira profissional na área de Enfermagem, essa universidade foi a que mais chamou atenção pelo currículo que possui,sendo muito semelhante ao da universidade da Colômbia (UCM). A UNIRIO cria capacidade para atividades de pesquisa, ensino e possui uma ampla variedade de campos em hospitais do Rio de Janeiro, o que proporciona um significativo impulso de aprendizado em diferentes situações e realidades. 
Uma vivência Brasil-Colombia em estágio de Enfermagem

\section{RESULTADOS}

As atividades visaram à melhoria da qualidade da assistência, da organização e eficiência da enfermaria pediátrica do referido hospital. Com a hospitalidade dos estudantes e profissionais e interesse das partes e nacionalidades envolvidas, para trabalhar em conjunto, o desempenho do grupo de estudantes de Enfermagem se mostrou bastante proveitoso. Conseguiram realizar todas as tarefas apresentadas, seja individualmente ou em grupo, obtendo êxito e reconhecimento dos profissionais.

Devido à amizade estabelecida e à disposição de todos os envolvidos, os estudantes conseguiram realizar mais do que era esperado inicialmente, o que resultou em dois trabalhos acadêmicos e um minicurso de Primeiros Socorros em Pediatria, para os alunos de Enfermagem do $6^{\circ}$ período que estavam em atividade prática na mesma enfermaria.

\section{CONSIDERAÇÕES FINAIS}

Após convívio quase diário entre os estudantes das duas nacionalidades, foi proporcionado a todos maior conhecimento das respectivas línguas,assim como também das culturas e diferenças dos sistemas de saúde e ensino, principalmente na atuação em Enfermagem. Os envolvidos puderam perceber que, apesar dos contrastes e das diferenças, devido à empatia e interesse do grupo em acolher e aprender, o contato foi benéfico, produtivo e agradável. A presente experiência de intercâmbio internacional na fase de Estágio Obrigatório na graduação em Enfermagem permitiu trocas e aprendizados significativos para os estudantes e outros profissionais, considerando-se principalmente os protocolos de Saúde tanto da Colômbia, quanto do Brasil, o que sempre gera conhecimento de novas práticas e, futuramente, pode vir a ser abordado e aprofundado em publicações da área.

\section{REFERÊNCIAS}

BRASIL. Lei No 9.394, de 20 de Dezembro de 1996. Estabelece as diretrizes e bases da educação nacional. Brasilia: Casa Civil, [1996]. Disponível em: http://www.planalto.gov.br/ccivil 03/leis/19394.htm

CAMPOS, A. C. Relação Comercial Brasil-Colômbia está aquém do potencial, dizem especialistas. Agência Brasil, out. 2015. Disponível online em: http://agenciabrasil.ebc.com.br/politica/noticia/2015-10/relacao-comercial-brasil-colombiaesta-aquem-do-potencial-dizem 
Uma vivência Brasil-Colombia em estágio de Enfermagem

CARLSON, E.; et al. Nursingas universal and recognisable: Nursing students perceptions of learning outcomes from intercultural peer learning webinars: A qualitative study. Nurse Education Today, v. 57, p. 54-59, 2017.

COLOMBIA, Ministerio de Salud y Protección Social. Plan de Beneficios em Salud. Sistema de seguridad social. Bogotá, Capital de Colombia; 2019.

Escola Profissional de Enfermeiros e Enfermeiras. Dicionário Histórico-Biográfico das Ciências da Saúde no Brasil (1832-1930). Fiocruz,. Disponível em: http://www.dichistoriasaude.coc.fiocruz.br/iah/pt/verbetes/escproenf.htm . Acesso em 20 out. 2019.

GUSKUMA, E. M.; et al. Mobilidade acadêmica internacional na formação em enfermagem: relato de experiência. Revista Brasileira de Enfermagem, v. 69, n. 5, p. 986-990, 2016.

JUNG, B. C.; et al. Colômbia: cenário de estudo em enfermagem no programa de mobilidade acadêmica internacional. Revista de Enfermagem da UFSM, v. 5, n. 4, p. 675-682, out./dez. 2015.

Sistema Único de Saúde (SUS): estrutura, princípios e como funciona. Ministério da Saúde. Disponível em: http://www.saude.gov.br/sistema-unico-de-saude .Acesso em 20.out.2019.

Recebido em: 21/11/2019

Aceito em: 10/12/2020 